\title{
Editorial: Emergent Effects of Noise in Biology: From Gene Expression to Cell Motility
}

\author{
Luis Diambra $^{1 *}$ and Moisés Santillán ${ }^{2}$ \\ ${ }^{1}$ Centro Regional de Estudios Genómicos, Universidad Nacional de La Plata-CONICET, La Plata, Argentina, ${ }^{2}$ Centro de \\ Investigación y de Estudios Avanzados del IPN, Unidad Monterrey, Ciudad Apodaca, Mexico
}

Keywords: cell motility, intinsic noise, extrinsic noise, gene experession, calcium signaling

\author{
Editorial on the Research Topic
}

Emergent Effects of Noise in Biology: From Gene Expression to Cell Motility

Deterministic modeling coarse-grains unitary events into population behavior, however this approach often misses the diversity of responses in cellular and molecular systems, due to long-time effects of stochastic fluctuations. The origins of stochasticity in biological systems are multiple and it is now generally accepted that this stochasticity can play key roles in emergent biological function at many scales: from intracellular processes to populations of macroscopic individuals. One can distinguish two sources: intrinsic fluctuations related to molecular processes, as those involved in biochemical reactions (in many instances the count of molecules participating in these processes is low, and so their stochastic nature becomes apparent); and extrinsic fluctuations due to random changes in the environment surrounding affecting the time evolution of the system.

Taking into account stochastic behavior is often a difficult but mandatory step to refine the description of a cellular and molecular behavior and to reveal the physiological functions. Thus, in the middle of several innovative approaches, stochastic modeling is being increasingly pursued in the context of modern statistical biophysics and cell biology.

Stochastic fluctuations associated to emerging cellular functions attracted the attention of scientists from various fields as diverse as physics, cell biology, applied mathematics and biophysics. Modeling both intrinsic and extrinsic random fluctuations is a unifying factor that links the articles of this Research Topic, which focus on three issues: (i) mathematical methods for studying the stochastic aspects of the gene expression; (ii) emerging role of noise in developmental and cellular processes, and (iii) information transmission, both in signaling pathways, as well as in the context of evolution. Bellow, each of the present Research Topic papers is briefly discussed.

Gonze et al. present a review of different modeling approaches that explore biological systems' robustness to noisy fluctuations. They first describe how a population of molecular clocks subject to intrinsic noise can work synchronously to produce sustainable circadian oscillations at the tissue level. Thus, despite the phase diffusion of the individual oscillators, intercellular coupling can induce robust oscillations even in the absence of an external cues [1]. Computational models that study intrinsic-noise effects on cell cycle oscillations are also reviewed, emphasizing the role of positive feedback in the oscillatory dynamics of the cyclin-dependent kinase network [2]. In another example, Gonze et al. review how calcium-induced calcium release converts stochastic $\mathrm{Ca}^{2+}$ dynamics into a more regular pattern [3], and mitochondrial buffering can counteract this regularity [4]. In the following work of this special issue, Givre et al. present a theoretical analysis of the information transmitted for the role of intracellular calcium dynamics. This study suggests that slow recovery from calcium release inhibition allows higher information transmission Givre et al. 
Information transmission, although not a new concept, is gaining momentum in cell signaling and gene network studies, and applications of this discipline are achieving unprecedented growth in several areas $[5,6]$. The review article from Pedraza et al. focuses on the relationship between fitness and information in biology. This study explores the adaption to changing environments in intermediate timescales and the quantification of the amount of information a cell needs to obtain from its environment through a theoretical approach.

Noise can also play a crucial role in embryonic development by acting as a trigger to determine the trajectory of cell differentiation due to cell multistability [7, 8]. In this sense, the review from Gonze et al. discuss several computational approaches that emphasize the interplay between noise and multistability during cell differentiation. Also in the context of development and cell differentiation in multicellular organisms, Holloway addresses the effect of the gene regulation variability, focusing on spatial patterning in Drosophila. The contribution also summarizes recent experimental advances in RNA transcripts quantification in fixed samples and, visualization of gene transcription in live embryos. The article gives some examples about noise regulatory mechanisms.

Cell size often exhibits variability from cell to cell and from generation to generation. Cellular size may be studied by either following single-cells over several generations or examining population data. The original research article by Thomas provides analytical computations for the size distribution of single cells, both in forward and backward lineages, and also across all births in a population tree. Their results highlight the differences between cell growth and division in isolation and in a population, and confirms that the extrapolation of results from isolated cells to population cells is not straightforward.

Cell migration is the result of several processes which involve the machinery of polarization and motility. When the direction of polarization is maintained during cell movement it develops

\section{REFERENCES}

1. Diambra L, Malta CP. Modeling the emergence of circadian rhythms in a clock neuron network. PLoS ONE. (2012) 7:e33912. doi: 10.1371/journal.pone.0033912

2. Barik D, Ball DA, Peccoud J, Tyson JJ. A stochastic model of the yeast cell cycle reveals roles for feedback regulation in limiting cellular variability. PLoS Comput Biol. (2016) 12:e1005230. doi: 10.1371/journal.pcbi.1005230

3. Guisoni N, Ferrero P, Layana C, Diambra L. Abortive and propagating intracellular calcium waves: analysis from a hybrid model. PLoS ONE. (2015) 10:e0115187. doi: 10.1371/journal.pone.0115187

4. Wacquier B, Romero Campos HE, González-Vélez V, Combettes L, Dupont G. Mitochondrial $\mathrm{Ca}^{2+}$ dynamics in cells and suspensions. FEBS J. (2017) 284:4128-42. doi: 10.1111/febs.14296

5. Pedraza JM, van Oudenaarden A. Noise propagation in gene networks. Science. (2005) 307:1965-9. doi: 10.1126/science.1109090

6. Monteoliva D, Diambra L. Information propagation in a noisy gene cascade. Phys Rev E. (2017) 96:012403. doi: 10.1103/PhysRevE.96.012403

7. Tosenberger A, Gonze D, Bessonnard S, Cohen-Tannoudji M, Chazaud C, Dupont G. A multiscale model of early cell lineage specification a persistent movement. This kind of active movement has been observed both in response to chemotactic gradients, as well as in the absence of external signals, and induce strong spatial and temporal correlations [9]. Guisoni et al. study the effects of density culture and additional correlations using cellular Potts models, as well as their importance for cell motility. Their work also summarizes current knowledge on modeling persistent cell motion. Further, Gosztolai et al. introduce a model for motile organisms which combines random exploration and finite perception that presents efficient search.

Finally, adequate mathematical tools and methodologies are critical for ensuring robust and reliable model predictions of stochastic gene networks. Methods are used to compute the time-dependent multivariate distributions and to describe the stochastic dynamics of gene networks. These methods are presented in an original research article of this Research Topic Innocentini et al. presents a meticulous dissection of three mathematical methods based on piecewise-deterministic approximations of the master equation applied to small genetic circuits. This analysis can be a useful reference for mathematical biologist and theoretician interested in models of stochastic gene expression.

We hope this Research Topic will stimulate further studies about the role of stochastic fluctuations on molecular and cellular biology.

\section{AUTHOR CONTRIBUTIONS}

LD and MS conceived and wrote the editorial.

\section{ACKNOWLEDGMENTS}

We would like to thank all reviewers for their constructive comments in the articles of this special issue. MS acknowledges financial support by Conacyt-México under grant FC-1132.

including cell division. NPJ Syst Biol Appl. (2017) 3:16. doi: 10.1038/s41540-0 17-0017-0

8. Guisoni N, Martinez-Corral R, Garcia Ojalvo J, de Navascués J. Diversity of fate outcomes in cell pairs under lateral inhibition. Development. (2017) 144:1177-86. doi: 10.1242/dev.137950

9. Marée AFM, Jilkine A, Dawes A, Grieneisen VA, Edelstein-Keshet L. Polarization and movement of keratocytes: a multiscale modelling approach. Bull Math Biol. (2006) 68:1169-211. doi: 10.1007/s11538-0 06-9131-7

Conflict of Interest Statement: The authors declare that the research was conducted in the absence of any commercial or financial relationships that could be construed as a potential conflict of interest.

Copyright (C) 2019 Diambra and Santillán. This is an open-access article distributed under the terms of the Creative Commons Attribution License (CC BY). The use, distribution or reproduction in other forums is permitted, provided the original author(s) and the copyright owner(s) are credited and that the original publication in this journal is cited, in accordance with accepted academic practice. No use, distribution or reproduction is permitted which does not comply with these terms. 